\title{
The Photometric System of Tsinghua-NAOC 80-cm Telescope at NAOC Xinglong Observatory
}

\author{
Fang Huang ${ }^{1,2}$, Jun-Zheng Li $^{2}$, Xiao-Feng Wang ${ }^{2}$, Ren-Cheng Shang ${ }^{2}$, Tian-Meng Zhang ${ }^{3,4}$, \\ Jing-Yao $\mathrm{Hu}^{3}$, Yu-Lei Qiu ${ }^{3}$ and Xiao-Jun Jiang ${ }^{3,4}$ \\ 1 Department of Astronomy, Beijing Normal University, Beijing, 100875, China; \\ hfbnu111@gmail.com \\ 2 Physics Department and Tsinghua Center for Astrophysics, Tsinghua University, Beijing, 100084, \\ China; \\ ${ }^{3}$ National Astronomical Observatories, Chinese Academy of Sciences, Beijing, 100012, China; \\ ${ }^{4}$ Key Laboratory of Optical Astronomy, National Astronomical Observatories, Chinese Academy of \\ Sciences, Beijing, 100012, China
}

\begin{abstract}
Tsinghua-NAOC (National Astronomical Observatories of China) Telescope (hereafter, TNT) is an 80-cm Cassegrain reflecting telescope located at Xinglong Observatory of NAOC, with main scientific goals of monitoring various transients in the universe such as supernovae, gamma-ray bursts, novae, variable stars, and active galactic nuclei. We present in this paper a systematic test and analysis of the photometric performance of this telescope. Based on the calibration observations on twelve photometric nights, spanning the period from year 2004 to year 2012, we derived an accurate transformation relationship between the instrumental ubvri magnitudes and standard Johnson $U B V$ and Cousins $R I$ magnitudes. In particular, the color terms and the extinction coefficients of different passbands are well determined. With these data, we also obtained the limiting magnitudes and the photometric precision of TNT. It is worthwhile to point out that the sky background at Xinglong Observatory may become gradually worse over the period from year 2005 to year 2012 (e.g., $\sim 21.4$ mag vs. $220.1 \mathrm{mag}$ in the $\mathrm{V}$ band).
\end{abstract}

Key words: instrumentation: detectors — site testing — telescopes

\section{INTRODUCTION}

Tsinghua-NAOC telescope (TNT), the first professional telescope owned by a university in China, is an 80$\mathrm{cm}$ Cassegrain telescope made by APM-Telescopes 1 in Germany. This telescope is located at Xinglong Observatory of NAOC $\left(117^{\circ} 34^{\prime} 39^{\prime \prime} E, 40^{\circ} 23^{\prime} 40^{\prime \prime} N\right.$, with an elevation of $\left.\sim 830 \mathrm{~m}\right)$, jointly operated by Tsinghua University and NAOC Chinese Academy of Sciences since year 2004. The main sciences conducted with TNT in recent years are multi-color, photometric studies of supernovae (SNe, Wang et al.2008 \& 2009 \& 2012, Zhang et al. 2010 \& 2012), active galactic nuclei (AGN, Liu et al.2010, Zhai et al. 2011 $\&$ 2012), gamma-ray bursts (GRBs, Xin et al.2010 \& 2011). Other projects of this telescope involve photometric observations of binary stars ( $\mathrm{Li}$ et al. 2009, Yang et al. 2010, Yan et al. 2012, Fang et al. 2012) and variable stars (Wu et al. 2005 \& 2006, and Fu et al. 2009). However, an overall examination of the performance of the CCD photometric system on TNT is still absent.

Knowing the properties and performance of a telescope such as throughput, detection limit, and instrument response will be of great assistance to the observers in preparing their observation proposals. These

\footnotetext{
1 http://www.apm-telescopes.com
} 
parameters allow a better estimate of the exposure time and predict photometric precision for individual objects. We started a program to investigate the characteristics of the CCD photometric system on TNT. Relevant evaluations of the photometric system of the BATC 60/90-cm schmidt telescope and the 85-cm telescope at Xinglong Observatory of NAOC are available from Yan et al. (2000) and Zhou et al. (2009), which help the users better understand the performance of these facilities and work out a reasonable observing plan.

This paper is organized as follows: in Sect. 2, we describe briefly about the observation system of TNT. Then we present the test results about the CCD detectors in Sect. 3. The photometric calibration results are given in Sect. 4 The systematic performance of TNT is addressed in Sect. 5, and we make a summary in Sect.6

\section{OBSERVATION SYSTEM}

TNT is a f/10 'classical' cassegrain, equatorial reflector. This telescope has a parabolic primary mirror with an effective diameter of $0.80 \mathrm{~m}$, and a hyperbolic secondary mirror with an effective diameter of $0.26 \mathrm{~m}$. The pointing of TNT is relatively fast and accurate, with the maximal slew speed being up to 4 degrees per second. At latitudes larger than 25 degrees, the pointing accuracy is better than $30^{\prime \prime}$. The pointing drift without guide star tracking is less than $1^{\prime \prime}$ in 15 minutes. The main parameters of TNT are very similar to those of the Lulin One-meter Telescope (LOT; Kinoshita et al.2005), except for differences in the aperture of the main mirror and the cooling-down mode of the CCD detector.

The CCD detector mounted on TNT is Princeton Instruments VersArray:1300B 2 . This is a highperformance, full-frame digital camera system that utilizes a back-illuminated, scientific-grade CCD. With a $1340 \times 1300$ imaging array $(20 \times 20 \mu \mathrm{m} /$ pixel $)$, this system provides a field-of-view (FOV) of $11.5^{\prime} \times 11.2^{\prime}$ with a spatial resolution of $\sim 0.52^{\prime \prime}$ pixel $^{-1}$. The main parameters of the VersArray:1300B CCD are listed in Table 1 It has two readout modes, with the readout time being about 18 seconds in the slow mode (100 $\mathrm{KHz})$ and about 2 seconds in the fast mode (1 MHz). There are nominally three 1300B CCDs that have been used on TNT since the start of observation in 2004. The 1300B-1 CCD had been used before the year 2006, and later replaced by the 1300B-2 CCD during the period from 5 Jan. to 14 Jun. in year 2006 for maintenance. After that, the 1300B-3 CCD had been used until Sept. 2010 when it was broken, and the 1300B-1 was installed again on TNT as a replacement.

Table 1 Parameters of the VersArray:1300B.

\begin{tabular}{ll}
\hline Features & Specifications \\
\hline Pixel number & $1340 \times 1300$ \\
Pixel Size & $20 \mu \mathrm{m} \times 20 \mu \mathrm{m}$ \\
Imaging area & $26.8 \mathrm{~mm} \times 26 \mathrm{~mm}$ \\
Fill factor & $100 \%$ \\
AD conversion & $16 \mathrm{bits}$ \\
Scan rates & $100 \mathrm{kHz}, 1 \mathrm{MHz}$ \\
Full frame readout time & $18 \mathrm{~s} @ 100 \mathrm{kHz}, 1.8 \mathrm{~s} @ 1 \mathrm{MHz}$ \\
Read noise & $2.8 e^{-} @ 100 \mathrm{kHz}, 8 e^{-} @ 1 \mathrm{MHz}$ \\
Software-selectable gains & $1 / 2 \times, 1 \times, 2 \times$ \\
Dark current & $0.5-1 e^{-} p i x^{-1} h r^{-1}$ \\
Nonlinearity & $\leq 2 \%$ \\
Cooling medium & Liquid nitrogen \\
Operating temperature & $-110^{\circ} \mathrm{C}$ \\
Thermostating precision & $\pm 0.05^{\circ} \mathrm{C}$ \\
\hline
\end{tabular}

The filters used on TNT are manufactured by the Custom Scientific, Inc. (USA) 3 , which are the standard Johnson $U B V$ and Cousin $R I$ system (Bessell 1990). This has been indicated by a small color-term correction needed to transform the photometric results from the instrumental system of TNT to the standard $U B V R I$ system (e.g., Wang et al.2008\& 2009).

2 http://www.princetoninstruments.com

3 http ://www.customscientific.com/astroresearch.html 


\section{CCD TESTS}

\subsection{Bias Level}

A bias level is present in every CCD image, arising from an electronic offset which is added to the signal of the CCD before being converted to the digital values. Its stability has a non-negligible effect on the high precision photometry. The bias level has been measured for all the three CCDs used on TNT in both the slow and fast readout modes. We performed a continuous 30-hour test of the bias for the 1300B-1 CCD, an 8-hour test for the 1300B-2, and a 7-hour test for the 1300B-3. These results are reported in Table 2.

One can see from Fig. 1 that the mean bias level is related to the readout mode of the CCD. For the 1300B-1 CCD, this value is about 195 Analog-to-Digital Unit (ADU) for the slow mode and about 400 ADU for the fast mode. We note that the bias measured in the fast readout mode shows some fluctuations, which might be affected by the ambient environments such as the temperature. Further studies are needed to clarify this phenomenon. Owing to an instability of the bias level seen in the fast readout mode, the observers are suggested to take frequent bias frames during observations to achieve high precision photometry when this mode is used.

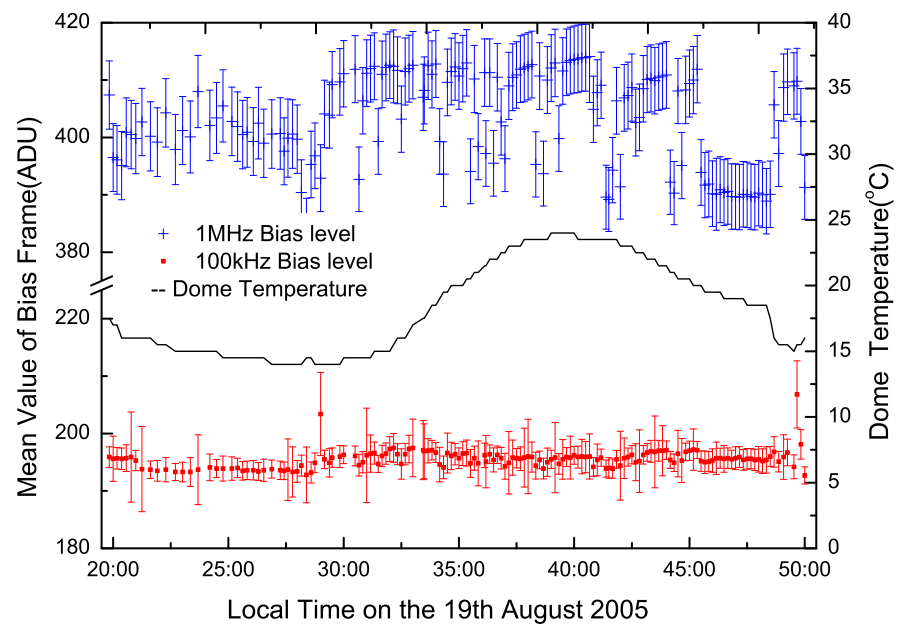

Fig. 1 The mean bias level of VersArray: 1300B-1 CCD derived in the slow and fast readout modes during a continuous 30 hour period. The solid curve represents the dome temperature during the measurements of the bias.

\subsection{Gain and Readout Noise}

The gain $(\mathrm{G})$ of a CCD is the ratio between the number of electrons recorded by the CCD chip and the counts of ADU contained in the CCD image. It is useful to know this value in order to evaluate the performance of the CCD camera. Knowledge of the gain allows the calculation of the readout noise $(\mathrm{R})$ and other quantities of the CCD. One can measure the gain of a CCD by comparing the signal level to the amount of variation in the signal, e.g., in the flatfield images. This works because the relationship between counts and electrons is different for the signal and the variance. The gain of a CCD can be determined from the following equation (Howell 2000):

$$
G=\frac{(\overline{F 1}+\overline{F 2})-(\overline{B 1}+\overline{B 2})}{\sigma_{F 1-F 2}^{2}-\sigma_{B 1-B 2}^{2}},
$$


Where $\overline{F 1}, \overline{F 2}$ are the mean values of different flatfield images, and $\overline{B 1}, \overline{B 2}$ represent those of the bias images. $\sigma_{F 1-F 2}^{2}$ and $\sigma_{B 1-B 2}^{2}$ are the standard deviation of the difference between two flatfield images and two bias images, respectively. Subtracting two flatfield images increases the noise by a factor of $\sqrt{2}$. Therefore, the correlation between the signal $S$ and the noise $N$ can be expressed as

$$
N=\sqrt{\frac{S}{G}+\left(\frac{R}{G}\right)^{2}}
$$

Here, $R$ is the readout noise.

We took twilight flatfield images in the $B$ band, with the exposure time varying from $0.1 \mathrm{~s}$ to $400 \mathrm{~s}$. At each time of exposure, we took four flatfield images in the slow readout mode and another four images in the fast readout mode. We then chose 2 better ones and performed the subtraction to determine the standard deviation $\sigma_{F}$. The noise level can be obtained by dividing the standard deviation by $\sqrt{2}$. To obtain a mean signal, we subtracted the combined bias frame from the flatfield images. The noise $\mathrm{N}$ and signal $\mathrm{S}$ measured from the flatfield images are used to determine the gain and readout noise through a best fit to the relation shown by equation (2). We also calculated these two parameters using the task FINDGAIN in IRAF 4 . Table 2 also lists the resultant gain and the readout noise derived for 1300B-1,2,3 CCDs used on TNT.

Table 2 Bias, gain, and readout noise determined for the VersArray: 1300B CCD attached to TNT. Two readout rates at $100 \mathrm{KHz}$ and $1 \mathrm{MHz}$ are indicated in the brackets of column (2).

\begin{tabular}{llccc}
\hline \hline & Readout Mode & $\begin{array}{c}1300 \mathrm{~B}-1 \\
\text { (before 2006.1) }\end{array}$ & $\begin{array}{c}1300 \mathrm{~B}-2 \\
(2006.1-6)\end{array}$ & $\begin{array}{c}1300 \mathrm{~B}-3 \\
\text { (after 2006.6) }\end{array}$ \\
\hline Bias(ADU) & Slow Mode(100kHz) & $195 \pm 3$ & $110 \pm 2$ & $185 \pm 2$ \\
& Fast Mode(1MHz) & $403 \pm 6$ & $182 \pm 4$ & $213 \pm 5$ \\
\hline \hline Readnoise $\left(e^{-}\right)$ & Slow Mode(100kHz) & $2.75 \pm 0.03$ & $2.90 \pm 0.05$ & $2.50 \pm 0.21$ \\
(findgain in IRAF) & Fast Mode(1MHz) & $9.31 \pm 0.34$ & $5.63 \pm 0.08$ & $5.94 \pm 0.33$ \\
\hline Readnoise $\left(e^{-}\right)$ & Slow Mode(100kHz) & $2.72 \pm 0.18$ & $2.92 \pm 3.90$ & - \\
(fit Signal \& Noise) & Fast Mode(1MHz) & $9.56 \pm 0.50$ & $6.16 \pm 3.79$ & - \\
\hline \hline Gain( $e^{-} /$ADU) & Slow Mode(100kHz) & $1.96 \pm 0.02$ & $1.90 \pm 0.03$ & $1.73 \pm 0.09$ \\
(findgain in IRAF) & Fast Mode(1MHz) & $2.22 \pm 0.05$ & $1.99 \pm 0.03$ & $1.81 \pm 0.07$ \\
\hline Gain( $e^{-} /$ADU) & Slow Mode(100kHz) & $1.99 \pm 0.01$ & $1.86 \pm 0.02$ & - \\
(fit Signal \& Noise) & Fast Mode(1MHz) & $2.23 \pm 0.01$ & $1.99 \pm 0.01$ & - \\
\hline \hline
\end{tabular}

\subsection{Linearity of the CCD Response}

One advantage of a modern CCD is its linear response over a large dynamic range. While some pixel values in the images may be unusable if they are saturated (due to that the charge exceeds the full well capacity) or are within the nonlinear range. To check the linear response of the VersArray:1300B CCD, we measured the ADU counts as a function of exposure time using the unfiltered flatfield images. For the 1300B-1 CCD, we use images taken on August 21, 2005 with the exposure time of $3 \sim 80 \mathrm{~s}$, while 1300B-2 on April 6, 2006 with the exposure time of $0.1 \sim 400 \mathrm{~s}$. Fig. 2 shows a relationship in both the fast and slow modes for the 1300B-1 and the 1300B-2 CCDs respectively. One can see that the linear correlation holds for the pixel value up to $\sim 50,000 \mathrm{ADU}$, with the correlation coefficients 0.9998 and 0.9997 respectively.

\subsection{Dark Current}

A routine step of processing the CCD images involves a subtraction of dark current. Dark current of a CCD usually originates from the collecting of electrons within the potential well of a pixel in the image, which can become part of the signal and is indistinguishable from the astronomical photons. It is usually specified

\footnotetext{
4 IRAF, the Image Reduction and Analysis Facility, is distributed by the National Optical Astronomy Observatory, which is operated by the Association of Universities for Research in Astronomy, Inc. (AURA) under cooperative agreement with the National Science Foundation(NSF).
} 

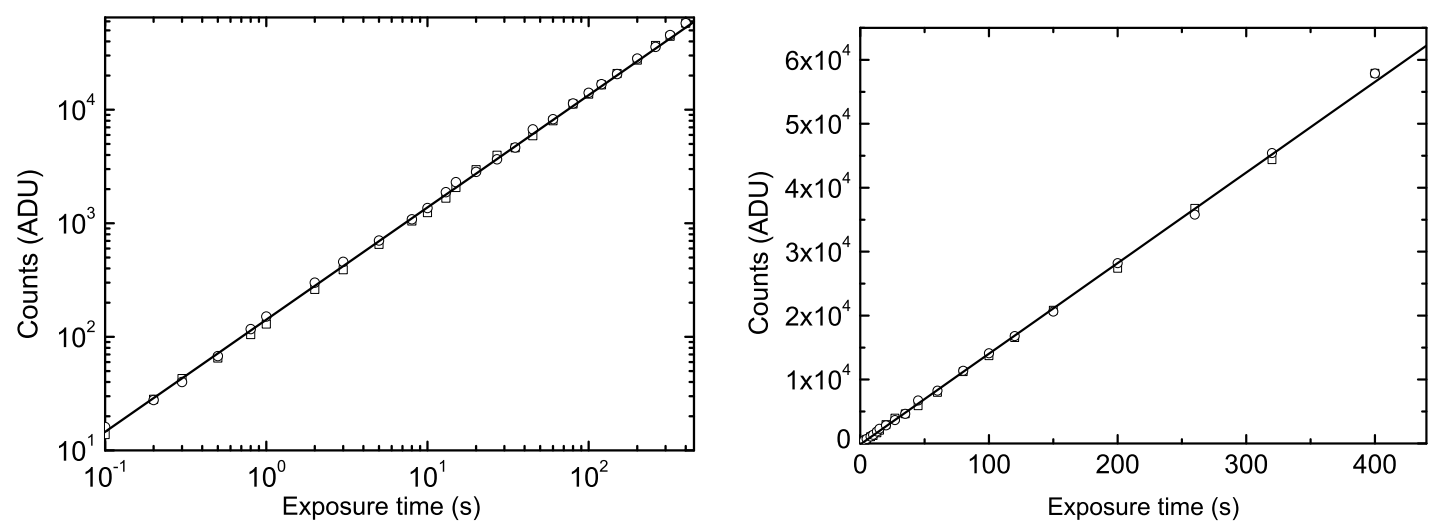

Fig. 2 ADU counts of the pixels in the flatfield images as a function of the exposure time for the 1300B-1 (left panel) and 1300B-2 (right panel). The counts in the fast mode are shown as the squares and those recorded in the slow mode are denoted by the circles. The straight lines show linear relationships in both modes.

as the number of thermal electrons generated per second per pixel or as the actual current generated per area of the device. The thermal dark current depends on the CCD operation temperature (see Figure 3.6 in Howell 2000 for a relation between the dark current and the temperature), which becomes nearly negligible for a properly cooled CCD.

Dark frames with different integration time (e.g., $600 \mathrm{~s}$ and $3600 \mathrm{~s}$ ) were obtained for the 1300B-1 CCD to estimate the dark current. We found that the mean dark current generate rate of the 1300B-1 is about $0.00025 e^{-} s^{-1}$ pixel $^{-1}$ for an operation temperature of $-120^{\circ} \mathrm{C}$. It is not surprising that the dark current level of the CCD on TNT is much lower than that of the CCD on LOT (i.e., 0.064e $e^{-} s^{-1}$ pixel $^{-1}$ ) since the working temperature of the latter is much higher with $\mathrm{T}=-50^{\circ} \mathrm{C}$. With the above dark current generation rate, we estimate that an observation with the exposure time of 600 s will produce a dark current of $0.15 e^{-} \mathrm{pixel}^{-1}$. This is far less than the signal and noise. Hence, we neglect such a minor effect in our image reduction.

\section{PHOTOMETRIC CALIBRATIONS}

The magnitudes obtained by TNT are the instrumental magnitudes. To compare our photometric results with those obtained from other instruments, we need to convert our instrumental magnitudes into the magnitudes defined in the standard $U B V R I$ system. To obtain this conversion, it is essential to know the transformation equations, which are usually expressed as:

$$
\begin{gathered}
u=U+Z_{U}+k_{U}^{\prime} X+C_{U}(U-B), \\
b=B+Z_{B}+k_{B}^{\prime} X+C_{B}(B-V), \\
v=V+Z_{V}+k_{V}^{\prime} X+C_{V}(B-V), \\
r=R+Z_{R}+k_{R}^{\prime} X+C_{R}(V-R), \\
\quad i=I+Z_{I}+k_{I}^{\prime} X+C_{I}(V-I) .
\end{gathered}
$$

where ubvri are the instrumental magnitudes, $U B V R I$ are the standard magnitudes, $Z_{U}, Z_{B}, Z_{V}, Z_{R}, Z_{I}$ are the zero point magnitudes, $k_{U}^{\prime}, k_{B}^{\prime}, k_{V}^{\prime}, k_{R}^{\prime}, k_{I}^{\prime}$ are the first-order extinction coefficients, $C_{U}, C_{B}, C_{V}, C_{R}, C_{I}$ are the color terms, and $X$ is the airmass. The above parameters can be simultaneously determined by observing a series of Landolt's standard stars covering a certain range of airmass and colors (Landolt 1992). 
Observations of Landolt's standard stars were conducted on twelve photometric nights, spanning the period from Oct. 2004 to Mar. 2012. Most of these photometric nights are moonless or crescent nights, with steady and cloudless sky. For a better comparison of these observations obtained at different time, we divided the photometric nights and the corresponding results into three epochs: Epoch 1 (2004-2005), Epoch 2 (2006-2007), and Epoch 3 (2011-2012). Table 3 lists part of the Landolt's standard stars that were observed during Epoch 3. The typical exposure time for these stars is $300 \mathrm{~s}$ in U, 60s in B, 40s in V, 20s in $\mathrm{R}$, and 20s in I. The photometric data of these Landolt's standard stars were reduced using the "apphot" package of IRAF. The deduced coefficients in the transformation equations ((3) - (7)) are shown in Table 4.

Table 3 The Landolt's standard stars used for the photometric calibration in year 2011 and 2012.

\begin{tabular}{cllcccccc}
\hline Star & $\alpha(2000)$ & $\delta(2000)$ & $\mathrm{V}$ & $\mathrm{B}-\mathrm{V}$ & $\mathrm{U}-\mathrm{B}$ & $\mathrm{V}-\mathrm{R}$ & $\mathrm{R}-\mathrm{I}$ & $\mathrm{V}-\mathrm{I}$ \\
\hline 92_263 & $00: 55: 40$ & $+00: 36: 23$ & 11.782 & 1.048 & 0.843 & 0.563 & 0.522 & 1.087 \\
93_317 & $01: 54: 38$ & $+00: 43: 11$ & 11.546 & 0.488 & -0.055 & 0.293 & 0.298 & 0.592 \\
$94 \_251$ & $02: 57: 46$ & $+00: 16: 18$ & 11.204 & 1.219 & 1.281 & 0.659 & 0.587 & 1.247 \\
$95 \_190$ & $03: 53: 13$ & $+00: 16: 39$ & 12.627 & 0.287 & 0.236 & 0.195 & 0.220 & 0.415 \\
96_83 & $04: 52: 59$ & $-00: 14: 22$ & 11.719 & 0.179 & 0.202 & 0.093 & 0.097 & 0.190 \\
$97 \_75$ & $05: 57: 55$ & $-00: 09: 07$ & 11.483 & 1.872 & 2.100 & 1.047 & 0.952 & 1.999 \\
$98 \_666$ & $06: 52: 10$ & $-00: 23: 12$ & 12.732 & 0.164 & -0.004 & 0.091 & 0.108 & 0.200 \\
100_280 & $08: 53: 36$ & $-00: 36: 24$ & 11.799 & 0.494 & -0.002 & 0.295 & 0.291 & 0.588 \\
101_413 & $09: 56: 15$ & $-00: 11: 44$ & 12.583 & 0.983 & 0.716 & 0.529 & 0.497 & 1.025 \\
103_626 & $11: 56: 47$ & $-00: 21: 47$ & 11.836 & 0.413 & -0.057 & 0.262 & 0.274 & 0.535 \\
104_598 & $12: 45: 17$ & $-00: 16: 41$ & 11.479 & 1.106 & 1.050 & 0.670 & 0.546 & 1.215 \\
105_815 & $13: 40: 04$ & $-00: 02: 19$ & 11.453 & 0.385 & -0.237 & 0.267 & 0.291 & 0.560 \\
106_1024 & $14: 40: 07$ & $+00: 01: 31$ & 11.599 & 0.332 & 0.085 & 0.196 & 0.195 & 0.390 \\
107_484 & $15: 40: 17$ & $-00: 21: 31$ & 11.311 & 1.237 & 1.291 & 0.664 & 0.577 & 1.240 \\
108_475 & $16: 37: 00$ & $-00: 35: 01$ & 11.309 & 1.380 & 1.462 & 0.744 & 0.665 & 1.409 \\
109_381 & $17: 44: 12$ & $-00: 20: 55$ & 11.730 & 0.704 & 0.225 & 0.428 & 0.435 & 0.861 \\
110_280 & $18: 43: 07$ & $-00: 04: 02$ & 12.996 & 2.151 & 2.133 & 1.235 & 1.148 & 2.384 \\
111_1965 & $19: 37: 42$ & $+00: 26: 30$ & 11.419 & 1.710 & 1.865 & 0.951 & 0.877 & 1.830 \\
112_250 & $20: 42: 27$ & $+00: 07: 25$ & 12.095 & 0.532 & -0.025 & 0.317 & 0.323 & 0.639 \\
113_260 & $21: 41: 49$ & $+00: 23: 39$ & 12.406 & 0.514 & 0.069 & 0.308 & 0.298 & 0.606 \\
114_750 & $22: 41: 45$ & $+01: 12: 30$ & 11.916 & -0.041 & -0.354 & 0.027 & -0.015 & 0.011 \\
RU_152E & $07: 27: 25$ & $-01: 58: 47$ & 12.362 & 0.042 & -0.086 & 0.030 & 0.034 & 0.065 \\
PG1047+003C & $10: 50: 18$ & $-00: 00: 21$ & 12.453 & 0.607 & -0.019 & 0.378 & 0.358 & 0.737 \\
PG2349+002 & $23: 51: 53$ & $+00: 28: 17$ & 13.277 & -0.191 & -0.921 & -0.103 & -0.116 & -0.219 \\
\hline
\end{tabular}

The photometric data obtained at different epochs seem to give a similar mean value of the relevant coefficients except for the magnitude zeropoints. The large difference in the magnitude zeropoint between Epoch 3 and the other two epochs are primarily related to the specific definition of the magnitude zeropoint, e.g., with an offset of $5.0 \mathrm{mag}$ in all of the $U B V R I$ bands. The mean atmospheric extinction coefficient 5 at Xinglong Observatory, obtained with the most recent data (e.g., Epoch 3), are $0.55 \pm 0.06$ in $U, 0.35 \pm 0.02$ in $\mathrm{B}, 0.24 \pm 0.02$ in $\mathrm{V}, 0.17 \pm 0.02$ in $\mathrm{R}$, and $0.09 \pm 0.02$ in I, respectively. Recently, Zhou et al. (2009) also examined the atmospheric extinction at Xinglong based on the observations with the 85-cm telescope. Their studies show that the first-order atmospheric extinction coefficient in the BVRI bands are $0.33 \pm 0.01,0.24 \pm 0.01,0.20 \pm 0.01$, and $0.07 \pm 0.01$, respectively, which are consistent with ours within the quoted errors. In Table 5, we also compared our results with two earlier estimates for the site given by Shi et al. (1998).

The color terms determined at the above three epochs are generally in accordance with each other, except in the $U$ band where the variation is likely related to the change of the CCD that directly determines the quantum efficiency and hence the profile of the instrumental response curve. The 1300B-1 CCD was used during the periods over Epoch 1 and Epoch 3, and the corresponding photometric system has a larger U-band color term; while the 1300B-2 CCD photometric system has a smaller value. Figure 3 shows the correlations between the Landolt colors (Landolt 1992) and the instrument colors of TNT transformed by equations ((3)-(7)). The Landolt standard stars observed on $31 \mathrm{Dec}, 2011$ are used for the plot. Fitting those data points in a linear fashion yields a slope that is very close to 1.0 , with an rms $<0.1 \mathrm{mag}$ in

\footnotetext{
5 The extinction coefficients are in unit of magnitude per airmass.
} 
Table 4 Transformation coefficients of zero-point magnitudes, first-order atmospheric extinction coefficients, and color terms in the $U B V R I$ bands, derived from the calibration data of 12 photometric nights.

\begin{tabular}{|c|c|c|c|c|c|}
\hline Date(ymd) & $Z_{U}$ & $Z_{B}$ & $Z_{V}$ & $Z_{R}$ & $Z_{I}$ \\
\hline 20041026 & $1.162 \pm 0.145$ & $-1.317 \pm 0.046$ & $-1.711 \pm 0.036$ & $-1.671 \pm 0.039$ & $-0.872 \pm 0.032$ \\
\hline 20041127 & & $-1.356 \pm 0.028$ & $-1.687 \pm 0.021$ & $-1.604 \pm 0.020$ & $-0.875 \pm 0.017$ \\
\hline 20050902 & & $-1.023 \pm 0.017$ & $-1.502 \pm 0.011$ & $-1.552 \pm 0.016$ & $-0.910 \pm 0.024$ \\
\hline Epoch1 mean & $1.162 \pm 0.048$ & $-1.232 \pm 0.019$ & $-1.633 \pm 0.014$ & $-1.609 \pm 0.016$ & $-0.886 \pm 0.014$ \\
\hline 20061221 & $-0.186 \pm 0.046$ & $-1.854 \pm 0.024$ & $-2.040 \pm 0.021$ & $-2.155 \pm 0.020$ & $-1.692 \pm 0.029$ \\
\hline 20070107 & $0.032 \pm 0.045$ & $-1.798 \pm 0.034$ & $-1.978 \pm 0.028$ & $-2.113 \pm 0.026$ & $-1.573 \pm 0.021$ \\
\hline 20070111 & $-0.042 \pm 0.061$ & $-1.770 \pm 0.025$ & $-1.935 \pm 0.020$ & $-2.048 \pm 0.017$ & $-1.586 \pm 0.024$ \\
\hline 20071212 & $-0.123 \pm 0.061$ & $-1.837 \pm 0.032$ & $-2.025 \pm 0.032$ & $-2.085 \pm 0.033$ & $-1.659 \pm 0.028$ \\
\hline Epoch2 mean & $-0.080 \pm 0.027$ & $-1.815 \pm 0.014$ & $-1.995 \pm 0.013$ & $-2.100 \pm 0.012$ & $-1.628 \pm 0.012$ \\
\hline 20111024 & $5.650 \pm 0.369$ & $3.535 \pm 0.082$ & $3.210 \pm 0.036$ & $3.231 \pm 0.019$ & $3.691 \pm 0.068$ \\
\hline 20111223 & $5.524 \pm 0.105$ & $3.620 \pm 0.016$ & $3.259 \pm 0.010$ & $3.304 \pm 0.008$ & $3.729 \pm 0.036$ \\
\hline 20111231 & $5.900 \pm 0.066$ & $3.646 \pm 0.049$ & $3.214 \pm 0.071$ & $3.350 \pm 0.040$ & $3.756 \pm 0.045$ \\
\hline 20120306 & & $3.900 \pm 0.126$ & $3.650 \pm 0.110$ & $3.650 \pm 0.119$ & $4.200 \pm 0.117$ \\
\hline 20120327 & & $3.982 \pm 0.035$ & $3.566 \pm 0.027$ & $3.595 \pm 0.029$ & $4.047 \pm 0.021$ \\
\hline Epoch3 mean & $5.691 \pm 0.078$ & $3.737 \pm 0.033$ & $3.380 \pm 0.028$ & $3.426 \pm 0.026$ & $3.885 \pm 0.030$ \\
\hline Date(ymd) & $k_{U}^{\prime}$ & $k_{B}^{\prime}$ & $k_{V}^{\prime}$ & $k_{R}^{\prime}$ & $k_{I}^{\prime}$ \\
\hline 20041026 & $0.699 \pm 0.095$ & $0.311 \pm 0.030$ & $0.211 \pm 0.023$ & $0.153 \pm 0.025$ & $0.069 \pm 0.020$ \\
\hline 20041127 & & $0.306 \pm 0.020$ & $0.201 \pm 0.014$ & $0.121 \pm 0.015$ & $0.089 \pm 0.011$ \\
\hline 20050902 & & $0.272 \pm 0.011$ & $0.184 \pm 0.008$ & $0.149 \pm 0.011$ & $0.092 \pm 0.016$ \\
\hline Epoch1 mean & $0.699 \pm 0.032$ & $0.296 \pm 0.012$ & $0.199 \pm 0.009$ & $0.141 \pm 0.010$ & $0.083 \pm 0.009$ \\
\hline 20061221 & $0.648 \pm 0.027$ & $0.295 \pm 0.014$ & $0.201 \pm 0.012$ & $0.152 \pm 0.011$ & $0.099 \pm 0.016$ \\
\hline 20070107 & $0.548 \pm 0.028$ & $0.295 \pm 0.020$ & $0.214 \pm 0.016$ & $0.175 \pm 0.015$ & $0.074 \pm 0.012$ \\
\hline 20070111 & $0.644 \pm 0.040$ & $0.332 \pm 0.016$ & $0.220 \pm 0.013$ & $0.158 \pm 0.011$ & $0.093 \pm 0.016$ \\
\hline 20071212 & $0.709 \pm 0.038$ & $0.307 \pm 0.020$ & $0.221 \pm 0.020$ & $0.158 \pm 0.021$ & $0.097 \pm 0.018$ \\
\hline Epoch2 mean & $0.637 \pm 0.017$ & $0.307 \pm 0.009$ & $0.214 \pm 0.008$ & $0.161 \pm 0.008$ & $0.091 \pm 0.008$ \\
\hline 20111024 & $0.548 \pm 0.242$ & $0.310 \pm 0.055$ & $0.180 \pm 0.021$ & $0.130 \pm 0.021$ & $0.104 \pm 0.053$ \\
\hline 20111223 & $0.602 \pm 0.147$ & $0.309 \pm 0.009$ & $0.220 \pm 0.006$ & $0.161 \pm 0.009$ & $0.089 \pm 0.024$ \\
\hline 20111231 & $0.510 \pm 0.053$ & $0.360 \pm 0.038$ & $0.316 \pm 0.026$ & $0.215 \pm 0.046$ & $0.120 \pm 0.030$ \\
\hline 0306 & & $0.447 \pm 0.086$ & $0.242 \pm 0.074$ & $0.180 \pm 0.078$ & $0.031 \pm 0.079$ \\
\hline 20120327 & & $0.314 \pm 0.025$ & $0.222 \pm 0.020$ & $0.153 \pm 0.020$ & $0.083 \pm 0.016$ \\
\hline Epoch3 mean & $0.553 \pm 0.058$ & $0.348 \pm 0.022$ & $0.236 \pm 0.017$ & $0.168 \pm 0.019$ & $0.085 \pm 0.021$ \\
\hline "Date(ymd) & $=C_{U}$ & $C_{B}$ & $C_{V}$ & cl $C_{R}$ & 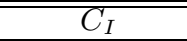 \\
\hline 20041026 & $-0.301 \pm 0.023$ & $-0.190 \pm 0.011$ & $0.077 \pm 0.009$ & $0.135 \pm 0.017$ & $-0.043 \pm 0.007$ \\
\hline 20041127 & & $-0.165 \pm 0.005$ & $0.083 \pm 0.004$ & $0.146 \pm 0.006$ & $-0.036 \pm 0.003$ \\
\hline 20050902 & & $-0.229 \pm 0.005$ & $0.067 \pm 0.004$ & $0.085 \pm 0.009$ & $-0.024 \pm 0.007$ \\
\hline Epoch1 mean & $-0.301 \pm 0.008$ & $-0.195 \pm 0.004$ & $0.076 \pm 0.003$ & $0.122 \pm 0.007$ & $-0.034 \pm 0.003$ \\
\hline 20061221 & $-0.132 \pm 0.016$ & $-0.132 \pm 0.008$ & $0.086 \pm 0.007$ & $0.110 \pm 0.011$ & $-0.037 \pm 0.009$ \\
\hline 20070107 & $-0.107 \pm 0.013$ & $-0.128 \pm 0.011$ & $0.076 \pm 0.009$ & $0.106 \pm 0.016$ & $-0.035 \pm 0.007$ \\
\hline 20070111 & $-0.136 \pm 0.011$ & $-0.134 \pm 0.006$ & $0.080 \pm 0.005$ & $0.105 \pm 0.007$ & $-0.040 \pm 0.005$ \\
\hline 20071212 & $-0.124 \pm 0.016$ & $-0.133 \pm 0.007$ & $0.079 \pm 0.007$ & $0.101 \pm 0.013$ & $-0.038 \pm 0.006$ \\
\hline Epoch2 mean & $-0.125 \pm 0.007$ & $-0.132 \pm 0.004$ & $0.080 \pm 0.004$ & $0.106 \pm 0.006$ & $-0.038 \pm 0.004$ \\
\hline 20111024 & $-0.316 \pm 0.063$ & $-0.144 \pm 0.011$ & $0.068 \pm 0.007$ & $0.108 \pm 0.007$ & $-0.026 \pm 0.011$ \\
\hline 20111223 & $-0.218 \pm 0.027$ & $-0.149 \pm 0.002$ & $0.064 \pm 0.004$ & $0.095 \pm 0.004$ & $-0.023 \pm 0.006$ \\
\hline 20111231 & $-0.367 \pm 0.034$ & $-0.146 \pm 0.010$ & $0.064 \pm 0.009$ & $0.076 \pm 0.015$ & $-0.025 \pm 0.007$ \\
\hline 20120306 & & $-0.164 \pm 0.035$ & $0.071 \pm 0.029$ & $0.088 \pm 0.055$ & $-0.015 \pm 0.031$ \\
\hline 20120327 & & $-0.155 \pm 0.008$ & $0.062 \pm 0.007$ & $0.083 \pm 0.014$ & $-0.033 \pm 0.005$ \\
\hline Epoch3 mean & $-0.300 \pm 0.015$ & $-0.152 \pm 0.008$ & $0.066 \pm 0.006$ & $0.090 \pm 0.012$ & $-0.024 \pm 0.007$ \\
\hline
\end{tabular}

different filter 6 . This means that transformation from the photometric system of TNT to the JohnsonCousion standard photometric system can be well established.

Note that the above color coefficients are obtained with normal stars with the $B-V$ color ranging from $-0.3 \mathrm{mag}$ to $+2.2 \mathrm{mag}$, and may not account for the whole photometric differences between the instrumental magnitudes and the standard Johnson-Cousion magnitudes for some variable sources such as $\mathrm{SNe}$ and GRBs because of their peculiar spectral shapes and features. Besides the color term correction,

${ }^{6}$ The scatter in $\mathrm{U}$ is slightly larger $(\sim 0.58 \mathrm{mag})$ due to relatively lower quality data. 
Table 5 Atmospheric extinction coefficients at Xinglong Observatory.

\begin{tabular}{cccccc}
\hline Year & $k_{B}^{\prime}$ & $k_{V}^{\prime}$ & $k_{R}^{\prime}$ & $k_{I}^{\prime}$ & References \\
\hline $2011-2012$ & $0.348 \pm 0.022$ & $0.236 \pm 0.017$ & $0.168 \pm 0.019$ & $0.085 \pm 0.021$ & 1 \\
2008 & $0.330 \pm 0.007$ & $0.242 \pm 0.005$ & $0.195 \pm 0.004$ & $0.066 \pm 0.003$ & 2 \\
$2006-2007$ & $0.307 \pm 0.009$ & $0.214 \pm 0.008$ & $0.161 \pm 0.008$ & $0.091 \pm 0.008$ & 1 \\
$2004-2005$ & $0.296 \pm 0.012$ & $0.199 \pm 0.009$ & $0.141 \pm 0.010$ & $0.083 \pm 0.009$ & 1 \\
1995 & 0.35 & 0.20 & 0.18 & 0.16 & 3 \\
1989 & 0.31 & 0.22 & 0.14 & 0.10 & 3 \\
\hline
\end{tabular}

REFERENCES: 1. this paper 2. Zhou et al. (2009) 3. Shi et al. (1998)
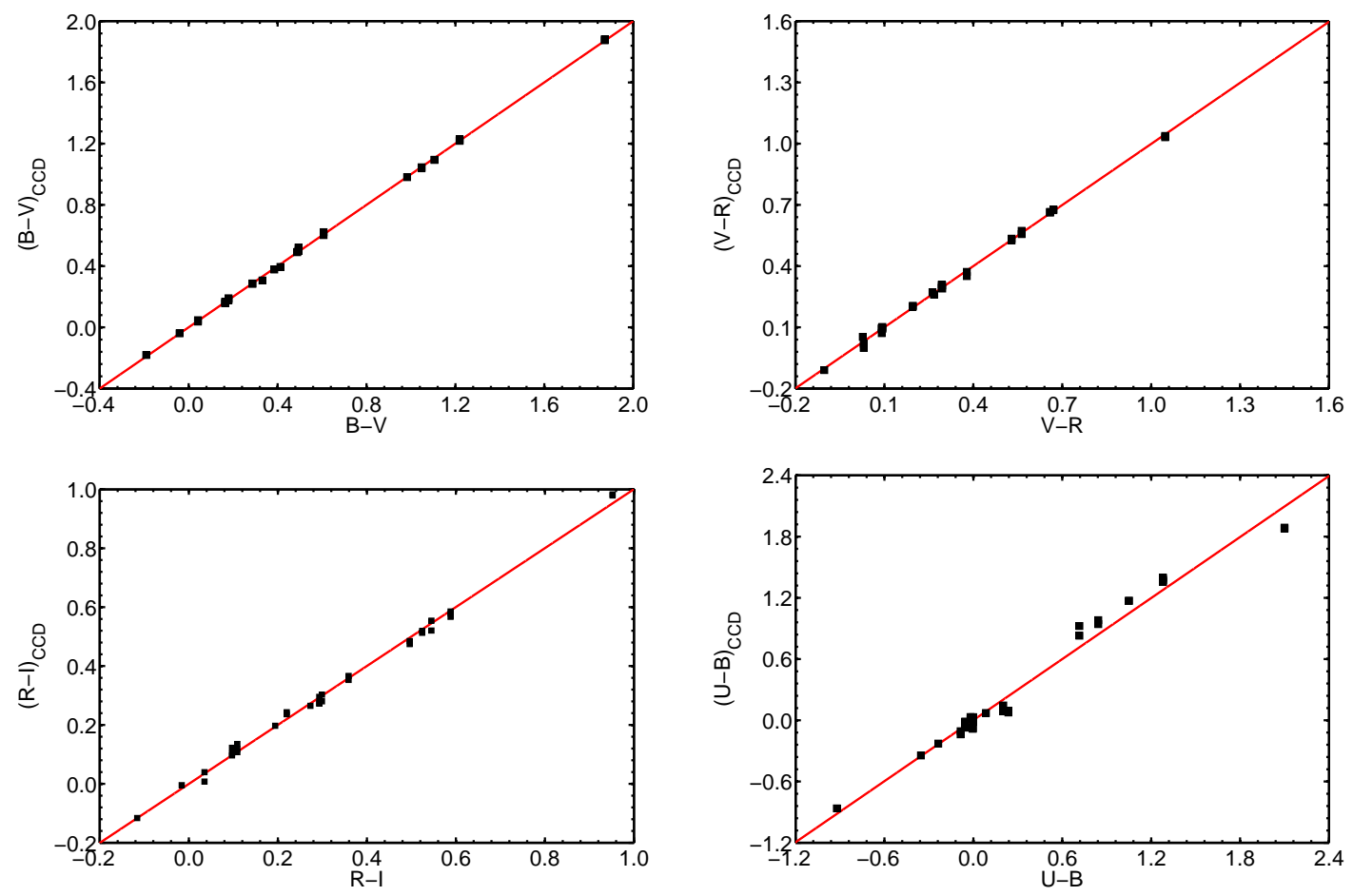

Fig. 3 The relationship between the Landolt (1992) color indices and the colors deduced from the transformation equations ((3)-(7)). The data taken on 31 Dec., 2011 are used for the plot.

additional corrections such as the S-corrections (Stritzinger et al. 2002) are usually required for precise photometry of these objects.

\section{SYSTEM PERFORMANCE}

\subsection{System Efficiency}

Using the photometric observations of the Landolt's standard stars, we could also estimate the total throughput of the overall observation system. This involves the filter response, the atmospheric transmission, the telescope optics and the detector quantum efficiency. Following the descriptions by Kinoshita et al. (2005) (see their Equations (15)-(18)), we computed the throughput efficiency of 1300B-1 CCD for the TNT observations. The results in different bands are summarized in Table 6, with higher throughput efficiency 
in $\mathrm{V}$ and $\mathrm{R}$ bands. This is in accordance with the CCD quantum efficiency light curves provided by the manufacturers.

Table 6 The total throughout of the TNT photometric system for the $U B V R I$ bands, including telescope optics, filter transmittance, and detector quantum efficiency.

\begin{tabular}{cccccc}
\hline Band & $\mathrm{U}$ & $\mathrm{B}$ & $\mathrm{V}$ & $\mathrm{R}$ & $\mathrm{I}$ \\
Throughput & $9.5 \%$ & $12.1 \%$ & $24.7 \%$ & $36.4 \%$ & $13.6 \%$ \\
\hline
\end{tabular}

\subsection{Sky Background Brightness}

As a byproduct of our photometric calibrations, we could also estimate the brightness of the night sky based on the flux of the sky background. The instrumental magnitudes were converted into the standard system with the transformation equations (3) - (7) and the coefficients shown in Table 3. We did not consider the effects caused by the difference in the airmass and the direction of the sky area 7 . As the sky background emission is affected significantly by the moon phase, we divided the twelve-night data into two groups: moonlit and moonless nights. During the moonless nights (2004 2007), the sky brightness was estimated to $\sim 21.8 \mathrm{mag}$ in $U, \sim 21.7 \mathrm{mag}$ in $B, \sim 21.2 \mathrm{mag}$ in $V, \sim 20.5 \mathrm{mag}$ in $R$, and $\sim 19.1 \mathrm{mag}$ in $I$, respectively.These values are generally consistent with the estimates obtained in year 1989 and year 1995 (Shi et al. 1998) (see also Table 7). The value in $V$ is also consistent with the mean value of the moonlight-corrected sky brightness derived from the BATC data(Liu et al. 2003). In year 2011, however, the sky brightness is found to be $\sim 21.3 \mathrm{mag}$ in $U, \sim 20.9 \mathrm{mag}$ in $B, \sim 20.0 \mathrm{mag}$ in $V, \sim 19.3 \mathrm{mag}$ in $R$, and $\sim 18.1 \mathrm{mag}$ in $I$, respectively. These values are apparently brighter than those obtained a couple of years ago, indicating that the sky background at Xinglong Observatory is becoming worse in recent years. This is perhaps related to the contamination of the city light of Beijing, Tianjin and Xinglong.

Table 7 The night sky background brightness at Xinglong Observatory. The brightness is expressed in the unit of mag $\operatorname{arcsec}^{-2}$.

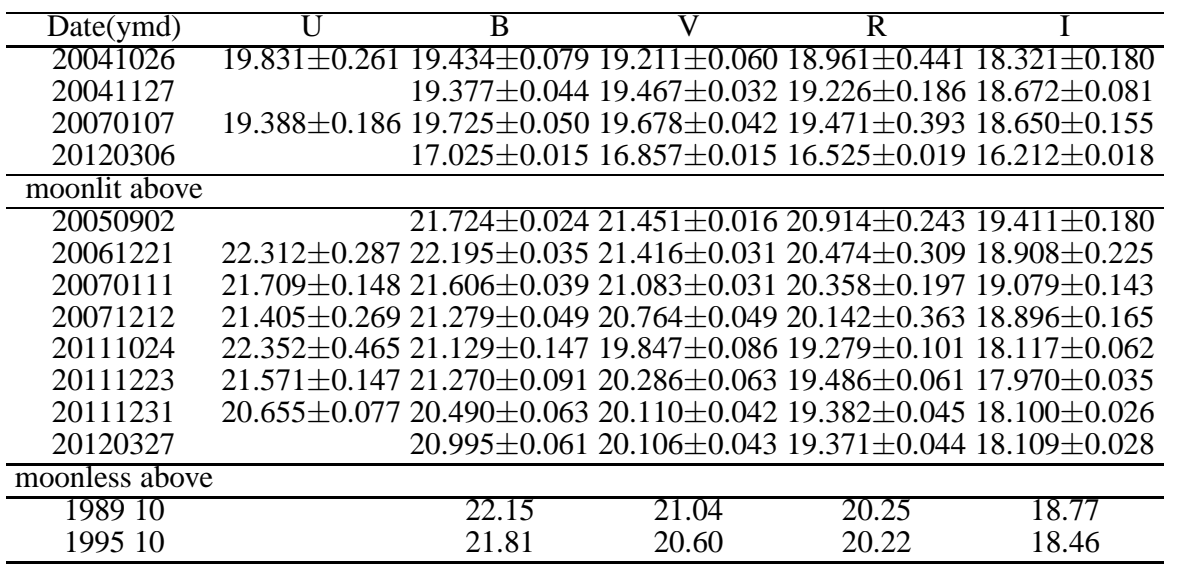

\footnotetext{
7 Xinglong Observatory is located on the northeast $115 \mathrm{~km}$ of Beijing, northeast $200 \mathrm{~km}$ of Tianjin, and east $7 \mathrm{~km}$ of Xinglong county. The city light from Beijing, Tianjin, and Xinglong can contribute significantly to the western sky background around Xinglong Observatory.
} 


\subsection{Limiting Magnitude and Photometric Precision}

We estimated the limiting magnitudes of the TNT photometric system as well. We used the equation below (Howell 2000) to perform our calculation:

$$
\frac{S}{N}=\frac{N_{\text {star }}}{\sqrt{N_{\text {star }}+n_{\text {pix }}\left(N_{\text {sky }}+N_{\text {dark }}+N_{\text {readout }}^{2}\right)}} .
$$

$N_{\text {star }}$ is the total number of photons collected from the targets. $N_{s k y}$ is the total number of photons per pixel from the sky background. $N_{\text {dark }}$ is the dark current per pixel from thermal electrons. $N_{\text {readout }}$ is the readout noise estimated in Section 2.2. $n_{\text {pix }}$ is the number of pixels under considerations for the calculation. The dark current electrons are neglected here.

The limiting magnitudes derived on the moonless nights with an aperture size of 6 arcsec in a slow readout mode are listed in Table 8 , with a mean value of $\mathrm{U} \sim 19.2 \mathrm{mag}, \mathrm{B} \sim 19.0 \mathrm{mag}, \mathrm{V} \sim 18.8 \mathrm{mag}, \mathrm{R} \sim 18.7$ mag, and I $\sim 18.2 \mathrm{mag}$, respectively, for a signal-to-noise ratio (SNR) of 100 and an integration time of $300 \mathrm{~s}$. Compared with detection limit for the $85-\mathrm{cm}$ telescope obtained by Zhou et al. (2009) (see their Table D.2), TNT seems to go slightly deeper, e.g. $18.8 \mathrm{mag}$ vs. $18.2 \mathrm{mag}$ in V for a 300 -s exposure, given a similar exposure time and SNR.

Table 8 Limiting magnitudes (for a signal-to-noise ratio of 100) derived in the slow mode using the data taken on the moonlit and moonless nights with an exposure time of $300 \mathrm{~s}$ and a photometric aperture of 6 arcsec.

\begin{tabular}{crrrrr}
\hline Date(ymd) & $\mathrm{U}$ & $\mathrm{B}$ & $\mathrm{V}$ & $\mathrm{R}$ & $\mathrm{I}$ \\
\hline 20041026 & $18.280 \pm 0.122$ & $18.091 \pm 0.038$ & $17.984 \pm 0.029$ & $17.863 \pm 0.215$ & $17.551 \pm 0.089$ \\
20041127 & & $18.064 \pm 0.021$ & $18.107 \pm 0.015$ & $17.992 \pm 0.090$ & $17.723 \pm 0.039$ \\
20070107 & $18.069 \pm 0.089$ & $18.230 \pm 0.024$ & $18.207 \pm 0.020$ & $18.109 \pm 0.189$ & $17.712 \pm 0.076$ \\
20111006 & & $17.795 \pm 0.021$ & $17.666 \pm 0.021$ & $17.889 \pm 0.020$ & $17.936 \pm 0.021$ \\
20120306 & & $17.577 \pm 0.007$ & $17.527 \pm 0.007$ & $17.744 \pm 0.009$ & $17.785 \pm 0.009$ \\
\hline moonlit mean & $18.175 \pm 0.030$ & $17.951 \pm 0.011$ & $17.898 \pm 0.009$ & $17.919 \pm 0.060$ & $17.741 \pm 0.025$ \\
\hline 20050902 & & $19.108 \pm 0.009$ & $19.000 \pm 0.007$ & $18.773 \pm 0.107$ & $18.080 \pm 0.087$ \\
20061221 & $19.321 \pm 0.092$ & $19.280 \pm 0.012$ & $18.985 \pm 0.013$ & $18.577 \pm 0.142$ & $17.838 \pm 0.109$ \\
20070111 & $19.102 \pm 0.056$ & $19.061 \pm 0.015$ & $18.846 \pm 0.013$ & $18.525 \pm 0.091$ & $17.920 \pm 0.069$ \\
20071212 & $18.981 \pm 0.107$ & $18.929 \pm 0.021$ & $18.707 \pm 0.022$ & $18.425 \pm 0.170$ & $17.832 \pm 0.080$ \\
20111024 & $19.353 \pm 0.017$ & $19.001 \pm 0.015$ & $18.697 \pm 0.016$ & $18.761 \pm 0.016$ & $18.452 \pm 0.016$ \\
20111223 & $19.257 \pm 0.008$ & $19.078 \pm 0.008$ & $18.865 \pm 0.009$ & $18.855 \pm 0.009$ & $18.410 \pm 0.010$ \\
20111231 & $19.116 \pm 0.006$ & $18.902 \pm 0.008$ & $18.772 \pm 0.007$ & $18.815 \pm 0.007$ & $18.474 \pm 0.007$ \\
20120327 & & $19.104 \pm 0.029$ & $18.902 \pm 0.021$ & $18.919 \pm 0.021$ & $18.582 \pm 0.013$ \\
\hline moonless mean $19.215 \pm 0.019$ & $19.058 \pm 0.006$ & $18.847 \pm 0.005$ & $18.706 \pm 0.033$ & $18.199 \pm 0.022$ \\
\hline
\end{tabular}

We further estimated the photometric precision of the TNT system. The errors of 1684 data points for the observations of 73 Landolt standard stars are shown in Fig. 4 . It is clearly seen that the photometric precision is $\leq 0.01 \mathrm{mag}$ for sources brighter than $15.0 \mathrm{mag}$, with an exposure time of 300-600 $\mathrm{s}$ in $U$, $60-120 \mathrm{~s}$ in $\bar{B}, 40-90 \mathrm{~s}$ in $V, 20-60 \mathrm{~s}$ in $R$, and 20-40 s in $I$, respectively. This is similar to the precision reached by the $85-\mathrm{cm}$ telescope for a similar exposure time and SNR (Zhou et al.2009).

\section{SUMMARY}

In this article, we evaluate performance of the VeryArray:1300B CCD photometric system mounted on the Tsinghua-NAOC 0.8-m telescope at Xinglong Observatory of NAOC. The evaluation results are summarized as follows:

(1) Typical CCD parameters such as the bias, gain, readout noise, and the dark current are derived for VeryArray:1300B. These parameters, especially the bias and the readout noise, are related to the readout modes. Compared with the fast readout mode, the slow mode produces an apparently lower bias level and readout noise. Because of a very low working temperature, the dark current of the CCD detector is very low and can be ignored in the image reduction. 

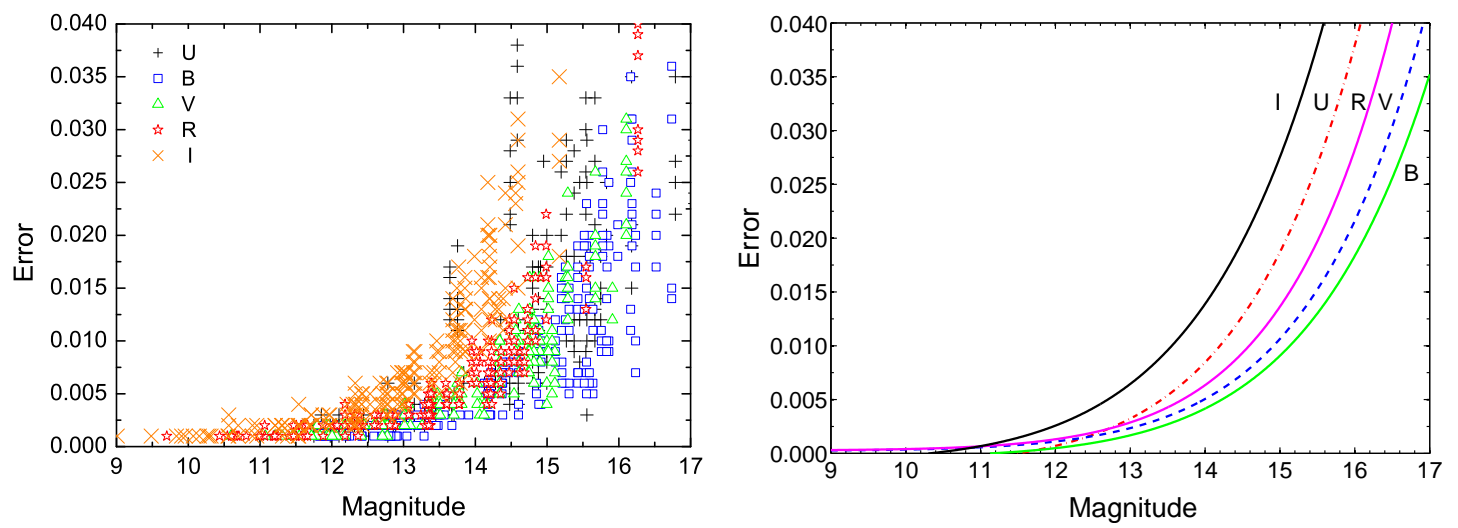

Fig. 4 Photometric errors of 73 Landolt standard stars obtained by the TNT system. Symbols in left panel show the distribution of the observed values in the $U B V R I$ filters while the curves in the right panel represent the best fit to the corresponding data points.

(2) Based on the observations of the Landolt's standard stars on a dozen of photometric nights, we derived the transformation coefficients between the instrumental ubvri magnitudes and the standard $U B V R I$ magnitudes: (i) the color terms used to normalize the photometry are relatively small for the ubvri filters mounted on TNT, suggesting that the response curves are similar to those of the standard Johnson/Cousins (Bessel) system; (ii) the atmospheric extinction coefficients in $U B V R I$ bands is robustly determined for the site of Xinglong with our extensive calibration data taken on the photometric nights.

(3) The limiting magnitudes are also obtained for TNT. With an exposure time of $300 \mathrm{~s}$, it can detect a point source with $\mathrm{B} \sim 19.0$ mag and $\mathrm{V} \sim 18.8$ mag for a SNR $\sim 100$.

(4) The emission of the sky background at Xinglong Observatory was also examined with our extensive calibration data, which shows an apparent increase after year 2005, e.g. from a level of $\mathrm{V} \sim 21.4$ mag in year 2005 to $\mathrm{V} \sim 20.2 \mathrm{mag}$ in year 2011. This change definitely brings a negative effect on the astronomical observations and researches at Xinglong Observatory.

Acknowledgments We thank the anonymous referee for his/her suggestive comments that help improve the manuscript. The work here is supported by the National Natural Science Foundation of China (NSFC grants 11178003, 11073013, and 10173003) and the National Key Basic Research Science Foundation (NKBRSF TG199075402).

\section{References}

Bessell, M. S., 1990, PASP, 102, 1181

Fang, X. S., Gu, S. H., et al., 2012, Research in Astron. Astrophys. (RAA), 12, 93

Fu, J. N., Zha, Q., et al., 2009, PASP, 121, 251

Howell, S. B., 2000, Handbook of CCD Astronomy (ISBN 0-521-64834-3), Cambridge University Press

Kinoshita, D., Chen, C.W., Lin, H.C., et al., 2005, ChJAA (Chin. J. Astron. Astrophys.), 5, 315

Landolt, A. U., 1992, AJ, 104, 340

Li, H. L., Yang, Y. G., Su, W., Wang, H. J., Wei,J. Y., 2009, Research in Astron. Astrophys. (RAA), 9, 1035

Liu, H., Wang, J., Mao, Y. F., Wei, J. Y., 2010, ApJ, 715, 113

Liu, Y., Zhou, X., Sun, W. X., et al., 2003, PASP, 115, 2003

Shi, H. M., Qiao, Q. Y., Hu, J. Y. et al., 1998, Acta Astrophysica Sinica, 18, 99

Stritzinger, M., Hamuy, M., et al., 2002, AJ, 124, 2100

Wang, X. F., Li, W. D., Filippenko, A. V. et al., 2008, ApJ, 675, 626 
Wang, X. F., Li, W. D., Filippenko, A. V. et al., 2009, ApJ, 697, 380

Wang, X. F., et al., 2012, in preparations

Wu, C., Qiu, Y. L., Deng, J. S., et al., 2005, AJ, 130, 1640

Wu, C., Qiu, Y. L., Deng, J. S., et al., 2006, A\&A, 453, 895

Xin, L.P., Zheng, W. K., et al., 2010, MNRAS, 401, 2005

Xin, L.P., Liang, E. W., et al., 2011, MNRAS, 410, 27

Yan, H. J., et al., 2000, PASP, 112, 691

Yan, J. Z., Li, H., Liu, Q. Z., 2012, ApJ, 744, 37

Yang, Y. G., Wei, J. Y., Kreiner, J. M., Li, H. L., 2010, AJ, 139, 195

Zhai, M., Zheng, W. K., Wei, J. Y., 2011, A\&A, 531, 90

Zhai, M., Wei, J. Y., 2012, A\&A, 538, A125

Zhang, T. M., Wang, X. F., et al., 2010, PASP, 122, 1

Zhang, T. M., et al., 2012, in preparations

Zhou, A. Y., Jiang, X. J., Zhang, Y. P., Wei, J. Y., 2009, Research in Astron. Astrophys. (RAA), 9, 349 